\title{
Picosecond Photophysics of 1,4-Diphenyl-butadiene in Condensed Phase. Solvent Assisted Electronic Level Inversion
}

\author{
C. RULLIERE, A. DECLEMY and Ph. KOTTIS
}

Centre de Physique Moléculaire Optique et Hertzienne, Laboratoire Associé au C.N.R.S. No. 283, Université de Bordeaux I, 351 Cours de la Libération, 33405 Talence, France

(Received October 17, 1984)

\section{INTRODUCTION}

The spectroscopic properties and photodynamics of polyenes have been extensively studied these last years. The reason is that these molecules are widely involved, or invoked as models, in photoisomerisation processes or in visual transduction.

In particular, the phenyl substituted polyenes have received specific attention because they fluoresce and that made them attractive systems for investigation with technics such as two-photon absorption spectroscopy ${ }^{1-3}$ and lifetime measurements with single photon techniques, ${ }^{4-7}$ both requiring reasonable fluorescence quantum yields.

An important controversial point in phenyl-polyenes is the ordering of the first singlet excited states, knowledge of which is fundamental for a better understanding of the photodynamics of these compounds. Indeed, the two first excited singlet states are of $B_{u}$ and of $A_{g}$ symmetry, in an order changing with the length of the polyenic chain. This ordering determines the allowed or forbidden character of the first transition $S_{0} \rightarrow S_{1}^{*}$ in one or two-photon absorptions and the subsequent dynamics of the excitation, when considering the totally symmetric ground state of $\boldsymbol{A}_{\mathrm{g}}$ symmetry. 
From theoretical calculations ${ }^{3}$ and experimental results obtained in two-photon spectroscopy and lifetime measurements, ${ }^{1,2,6}$ it has been deduced that in long polyenic chains $(n>2)$, the first singlet excited state $S_{1}^{*}$, is of $A_{g}$ symmetry. This conclusion allowed to explain previous discrepancies between observations and calculations, particularly that between the measured radiative lifetime and the calculated lifetime using the Strickler Berg relation. ${ }^{4}$ Moreover one photon spectroscopy in solutions, at room temperature, has shown that, owing to the small energy gap between $A_{g}$ and $B_{u}$ levels, the latter is also thermally repopulated. ${ }^{8,9}$

At the opposite limit of short chains $(n=1)$, state $S_{1}^{*}$ was given symmetry $B_{u}$ in the case of $t$-stilbene. This assignment, and extensive investigations of photoisomerisation processes in this compound, led to the elucidation of its photodynamics ${ }^{10}$ which are now well established. ${ }^{11}$

1,4-diphenyl-butadiene (DPB), with $n=2$, is an intermediate case. Because of quasi-resonant interactions between localized excitations in the phenyl groups and in the polyenic chain, strong solvent effects are expected in the photodynamics of this compound in solution; extensive studies have been carried out on recent years. On the one hand, in absorption data obtained by one or two-photon transitions from the ground state ( $\mathrm{A}_{g}$ symmetry), it has been shown that in glassy solutions $^{3}$ or in liquid solutions ${ }^{1,2}$ the state $S_{1}^{*}$ is of $A_{g}$ symmetry. More recently, Zewail and co-workers, ${ }^{12}$ using spectroscopy in a molecular beam, have shown that, as in a condensed phase, state $S_{1}^{*}$ in the isolated DPB molecule is of $A_{g}$ symmetry. On the other hand, investigations in emission spectroscopy of DPB molecules in liquid solutions, have shown that the fluorescing state, normally associated with the lower excited state $S_{1}^{*}$, is of $B_{u}$ symmetry. ${ }^{5,6}$ This attribution poses the difficult task of reconciling contradictory results of two series of experiments, based on absorption and emission spectroscopy, respectively.

More refined results, for the characterization of an excited state, have been reported by D. S. Kliger and co-workers in analyzing the absorption spectrum of excited DPB molecules in solution. ${ }^{13}$ In a comparative study of the absorption spectrum, $S_{1}^{*} \rightarrow S_{n}^{*}$, of DPB and of long chains $(n>2)$ of polyenes (where two absorption bands appear), they pointed out that the spectrum of DPB exhibits only one band as does the spectrum of $t$-stilbene. Then, on the basis of theoreti- 
cal calculations, they attributed $B_{u}$ symmetry to state $S_{1}^{*}$. Intending to reconcile these contradictory results, these authors suggested that the excited molecule undergoes fast reorganization: The most stable conformation of the ground state leads to an optically excited state of symmetry $B_{u}$, then fast reorganization of the molecule should lead to level inversion with a fluorescing state of symmetry $B_{u}$.

The purpose of the present paper is to deal with picosecond photodynamics associated with solvent assisted reorganization processes of excited DPB molecules in solution. Indeed, if reorganization processes lead to an inversion of the first excited singlet energy levels, then, the spectroscopic properties of the excited molecules must change with time after the optical excitation step; they must be very sensitive to the temperature and the viscosity of the solvent since these two parameters may assist or hinder such reorganization of the excited DPB molecule. However, in the time dependent emission, the spectral changes are not very sensitive in liquid solution, owing to the small energy gap between the two energy levels and to the broad bands overlapping which hides any structures. On the contrary, the time dependent absorption spectrum of excited molecules provides a very sensitive characterization of dynamics in the excited molecule. Indeed, levels inversion may cause drastic changes in the selection rules, with subsequent changes in the shape of the spectrum, according to whether the lower state $S_{1}^{*}$ evolves from $A_{g}$ to $B_{u}$ symmetry. To this respect, this paper presents a full analysis on a picosecond scale of the spectral evolution of the absorption shape of excited DPB molecules in solution, with various values of temperature and viscosity. The paper is divided in four sections: The picosecond technique is summarized in Section II; the experimental results on the excited populations are presented in Section III with various conditions of temperature and viscosity; interpretation and theoretical results are discussed in parallel in Section IV; concluding remarks on the performances and limitations of our method of investigation are presented in Section V.

\section{EXPERIMENTAL PICOSECOND TECHNIQUE}

\section{Experimental section}

Trans-1,4-diphenyl-1,3-butadiene (DPB) was obtained from Aldrich Chemicals and used without further purification since emission 
spectrum and lifetime were identical to the one described in the literature. ${ }^{3,5}$ Solvent used were all of spectroscopic grade and used also without further purification.

The experimental set-up used to obtain excited state spectra, as a function of time and temperature, has been described in detail elsewhere. ${ }^{14}$ It consists of a mode locked $\mathrm{Nd}^{3+} /$ YAG laser which delivers a single pulse of 25 ps duration.

The third $\left(28328 \mathrm{~cm}^{-1}\right)$ or the fourth $\left(37735 \mathrm{~cm}^{-1}\right)$ harmonics may be used as an excitation source of the sample to be studied. A picosecond continuum, generated in $\mathrm{D}_{2} \mathrm{O}$, and extending from 25000 to $10000 \mathrm{~cm}^{-1}$, crosses the sample and acts as a probe beam; It may be delayed with respect to the excitation pulse. The principle of this arrangement is to measure the absorption spectrum of the excited sample, as a function of time after the excitation, by measurement of the variations of the spectral distribution of the probe beam. The detector is an optical multichannel analyser (system DARSSTRACOR-NORTHERN TN 1710) using a photodiode array. Temperature control between $+60^{\circ} \mathrm{C}$ to $-100^{\circ} \mathrm{C}$ was insured using a SPECAC cryostat model $\mathrm{P} / \mathrm{N} 21000$ associated to an electronic temperature controller $\mathrm{P} / \mathrm{N} 20100$. The thermocouple is placed as near as possible to the sample to be studied, in order to get an accurate measurement of the temperature. With this apparatus, the temperature may be constant and stable within a range of $1^{\circ} \mathrm{C}$ over several hours, ensuring optimum experimental conditions for different temperatures.

\section{EXPERIMENTAL RESULTS ON PICOSECOND DYNAMICS OF EXCITED MOLECULES}

In four series of experiments, noted below (a)-(d), we probed by picosecond absorption spectroscopy, the influence of the temperature and of the intensity $I_{\mathrm{exc}}$ of the excitation beam, on the dynamics of the excited DPB molecule.

(a) $S_{1}^{*} \rightarrow S_{n}^{*}$ absorption spectra at room temperature: we studied the time evolution of the absorption spectrum of an excited assembly of DPB molecules dissolved in $\mathrm{MCH}$. Two typical spectra recorded at two different time delays after the excitation, at $t_{1}=30 \mathrm{ps}$ and at $t_{2}=100 \mathrm{ps}$, are shown on Figure 1. At room temperature the spectral behavior may be analyzed as follows: just after the excitation, the 


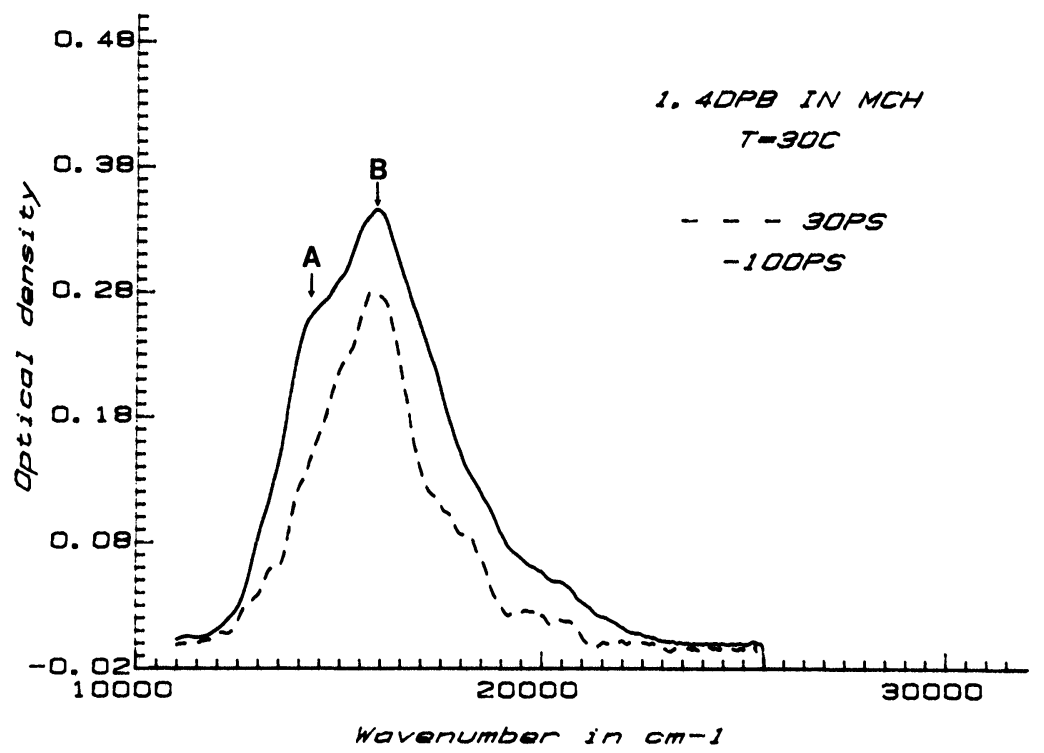

FIGURE 1. Excited DPB absorption spectrum at room temperature at two different times after excitation. Full line $t=30 \mathrm{ps}$. Dotted line: $t=100 \mathrm{ps}\left(c=5 \times 10^{-4} \mathrm{M}\right.$ in $\mathrm{MCH}$, $T=20^{\circ} \mathrm{C}$.

absorption spectrum of the excited entity is a single broad band centered at $16000 \mathrm{~cm}^{-1}$, that we call from here on band B. As time passes $(t>30 \mathrm{ps})$, a shoulder appears, on the red part of the spectrum, centered approximatively at $14500 \mathrm{~cm}^{-1}$, that we call from here on band $A$. The evolution kinetics of the principal band $B$ and of the shoulder (band $A$ ) are shown on Figure 2. The intensity of the principal band $\mathrm{B}$ develops quickly, reaching its maximum value at about 80 $100 \mathrm{ps}$; it decays with a lifetime of the order of $700 \mathrm{ps} \pm 100$. The latter is in agreement with values reported by other authors for the same kind of solvents. ${ }^{5,6}$ The intensity of the band A shows a slower rise-time, reaching its maximum value at about 100 ps; it decays with a lifetime similar to that of the principal band. The kinetics of band $A$ are not as accurate as those of the principal band $\mathrm{B}$, owing to the standard difficulty of extracting a weak band from a strong and broad band. However, we may unambiguously conclude from our data that, at room temperature, band $\mathrm{A}$ has a slower rise-time than band $\mathrm{B}$, and that it decays practically with an identical lifetime. 


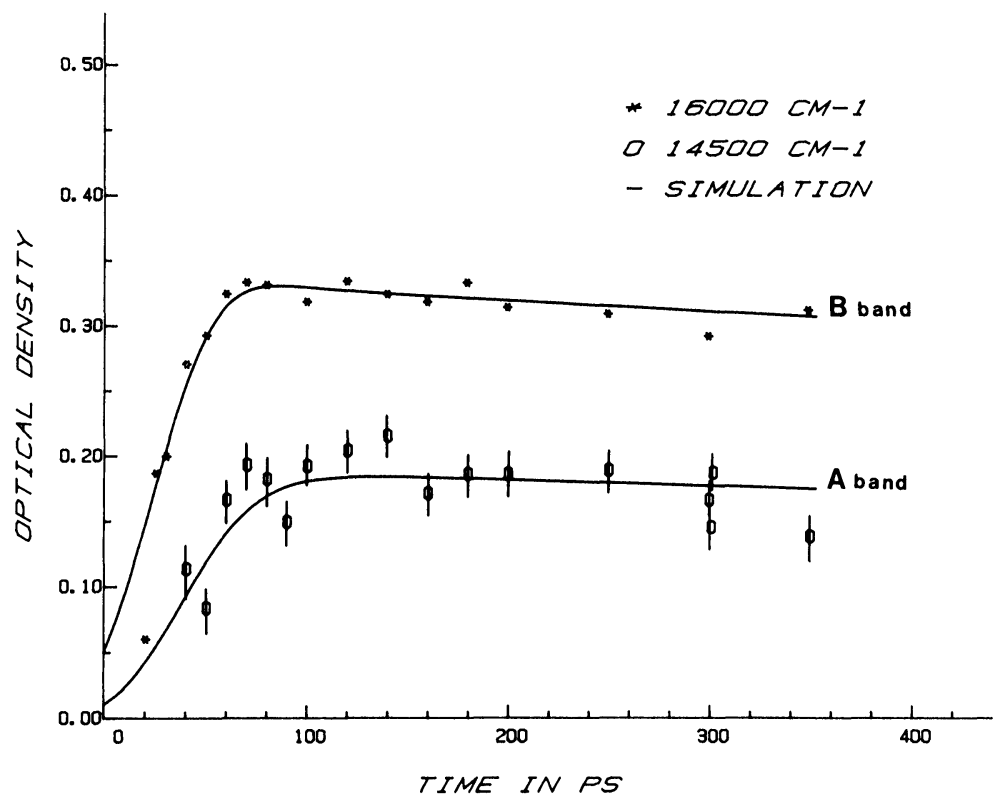

FIGURE 2 Kinetics evolution of the intensity of band B (*) at $16000 \mathrm{~cm}^{-1}$ and of band $A$ at $14500 \mathrm{~cm}^{-1}(O)$. Full lines are simulations of kinetics using parameters defined in the text (Scheme I).

(b) $S_{1}^{*} \rightarrow S_{n}^{*}$ absorption spectra at low temperatures: On Figure 3, we show the time evolution of the absorption spectrum recorded at low temperature $\left(-70^{\circ} \mathrm{C}\right)$; it presents the following characteristics: Just after the excitation $(t \sim 10 \mathrm{ps})$, the two bands $A$ and $B$ are now present, although the band $A$ is still the weak one. As time passes, its intensity increases drastically, it becomes comparable to that of band $B$ whose intensity decreases. At longer times $(t>50 \mathrm{ps})$ band B vanishes and the absorption spectrum reduces to a broad band $A$ with a lifetime of $1.2 \mathrm{~ns}$, similar to the value of the fluorescence lifetime of DPB, reported by other authors for non-polar solvent at low temperatures. ${ }^{5,6}$

(c) Thermal contribution in the absorption shape: We observed important temperature effects on the spectral shape. On Figure 4, we recorded the spectral changes, at fixed time delay $t=100 \mathrm{ps}$ after the excitation; large changes of the absorption spectrum are observed when the temperature is varied from $-80^{\circ} \mathrm{C}$ to $30^{\circ} \mathrm{C}$. At the lower and 


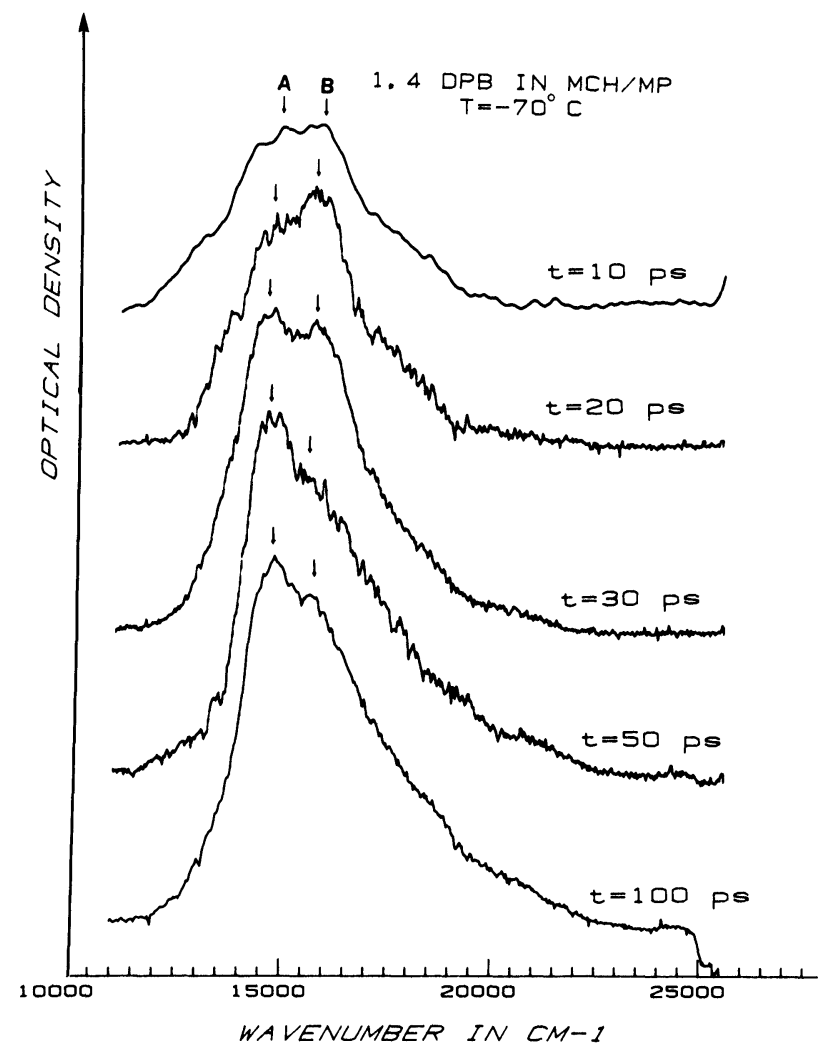

FIGURE 3 Temporal evolution of the spectrum of excited DPB at low temperatures $\left(c=5 \times 10^{-4} \mathrm{M}\right.$ and $\left.T=-70^{\circ} \mathrm{C}\right)$.

higher temperature limits, the absorption spectrum reduces predominantly to band A and to band B, respectively; at the intermediate values, when lowering the temperature, band $A$ increases at the expense of band $\mathrm{B}$.

(d) Spectral shape variation with the excitation intensity: We observed large changes of the absorption spectrum shape, which are not linearly dependent on the intensity $I_{\text {exc }}$ of the exciting beam. These anomalous variations are particularly important at low temperature, as it is illustrated on Figure 5, although they still persist, to a lesser extent at all temperatures, even at room temperature. These shape variations with $I_{\text {exc }}$ may be summarized in the following way, if we 


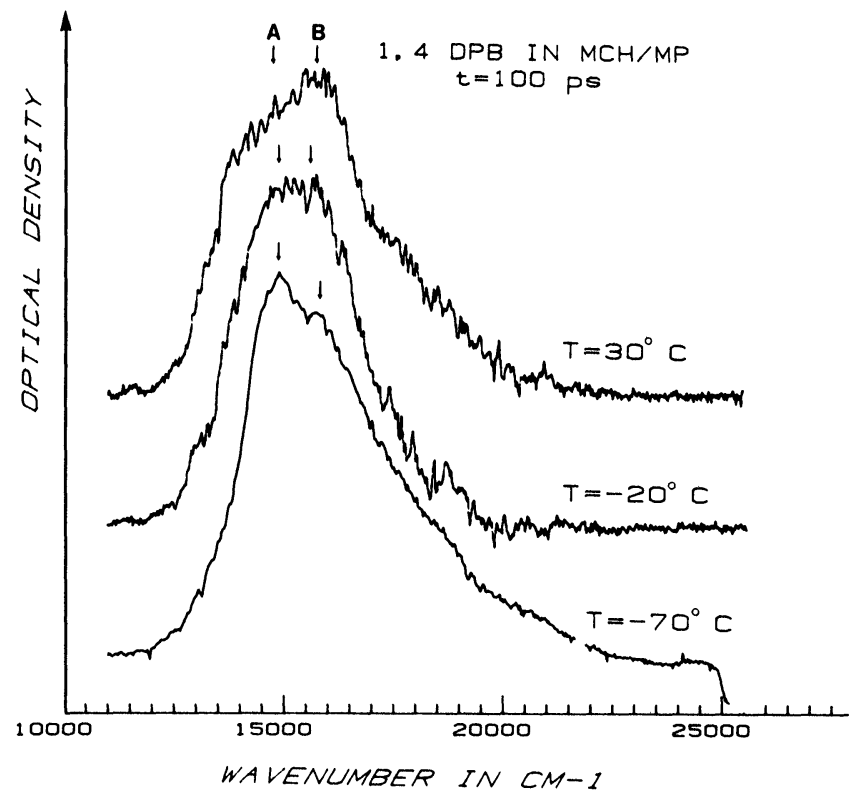

FIGURE 4 Spectral change of the excited DPB spectrum at different temperatures at fixed time $t=100 \mathrm{ps}$ after the excitation pulse $\left(c=5 \times 10^{-4} \mathrm{M}\right.$ in $\left.\mathrm{MCH} / \mathrm{MP}\right)$.

consider the intensity $I_{\text {exc }}$ to vary from 0 to 100 , in arbitrary units:

(i) in the range of low values of $I_{\text {exc }}\left(I_{\text {exc }}<30\right)$ no shape changes are observed with respect to the standard shape we discussed in our data (Figures 1-4): The shape of the absorption spectrum is not modified when the excitation intensity $I_{\text {exc }}$ varies in the range of low values.

(ii) In the range of high values of $I_{\text {exc }}\left(I_{\text {exc }}>30\right)$, the spectrum shape strongly depends on the value of $I_{\text {exc }}$ : The intensity of band B shows a quadratic increase with the intensity $I_{\text {exc }}$; it becomes predominant in the absorption spectrum at $t=100 \mathrm{ps}$ after the excitation step, as it is illustrated on Figure 5. On much higher excitation intensities saturation effects occur.

It is clear that the quadratic growth of band $\mathrm{B}$, in a certain $I_{\text {exc }}$ range, occurs at the expense of band $A$. For instance, at $T=-80^{\circ} \mathrm{C}$ and $t=100 \mathrm{ps}$, the quadratic growth makes band $\mathrm{B}$ predominant in the absorption spectrum, while it is not even observable at low values of $I_{\text {exc. }}$. 


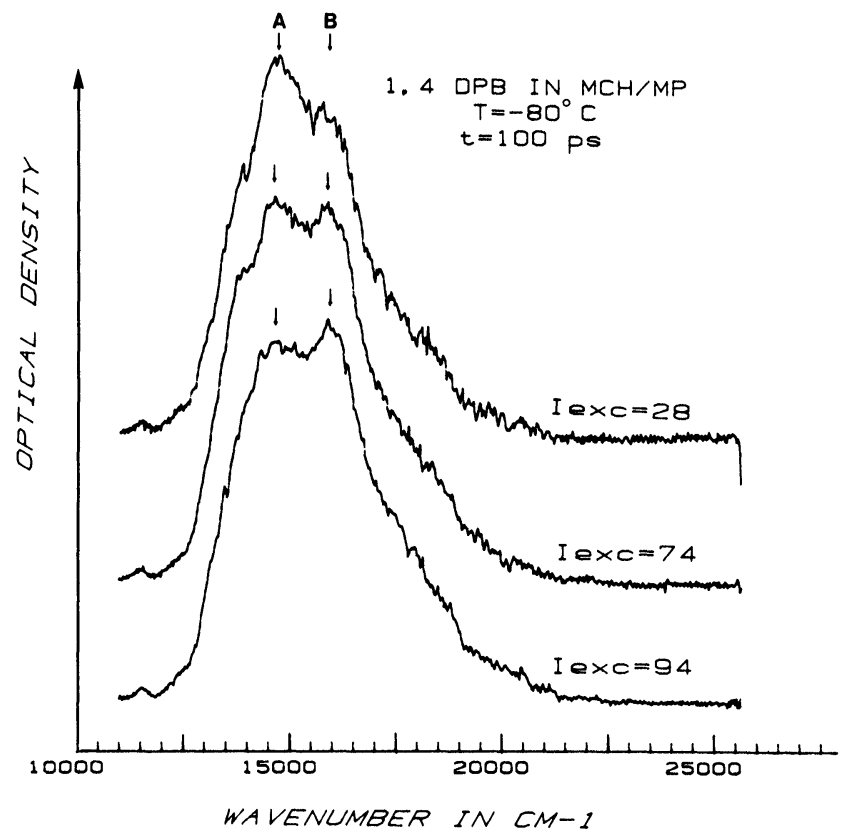

FIGURE 5 Evolution of the excited DPB spectrum at low temperature as a function of the excitation intensity. $\left(T=-80^{\circ} \mathrm{C}, c=5 \times 10^{-4} \mathrm{M}\right.$ in MCH/MP, $t=100 \mathrm{ps}, I_{\text {exc }}$ is in arbitrary units).

\section{SELF CONSISTENT MODEL INTERPRETATION OF THE RESULTS}

Our own results, as well as those reported by other authors, pose some questions on the photodynamics of the excited DPB molecule in solution, particularly on the level ordering of the two first excited singlet states of symmetry $B_{u}$ and $A_{g}$ and on their vibronic couplings, direct or solvent assisted.

These questions must be answered by the construction of a model which accounts self consistently for all the observations collected in a wide variety of experiments, such as absorption spectra of isolated molecules or absorption and emission spectra of molecules in solution, at various temperatures and viscosities. In this section, we want to show that a model involving the possibility of reorganization of the 
excited DPB molecule, with inversion of level ordering in solution, may account for all the experimental observations reported on DPB. Indeed, if we consider that in the ground state nuclear conformation, the lower excited singlet state is of the $A_{g}$ symmetry (and that the one photon optically excited state of symmetry $B_{u}$ lies above it) then solvent assisted conformational stabilization of the excited state may lead to level inversion with the $B_{u}$ state becoming the lower excited singlet state, cf. Figure 6 . This cycle of excitation may be sketched in the following way including the solvent contribution: In the ground
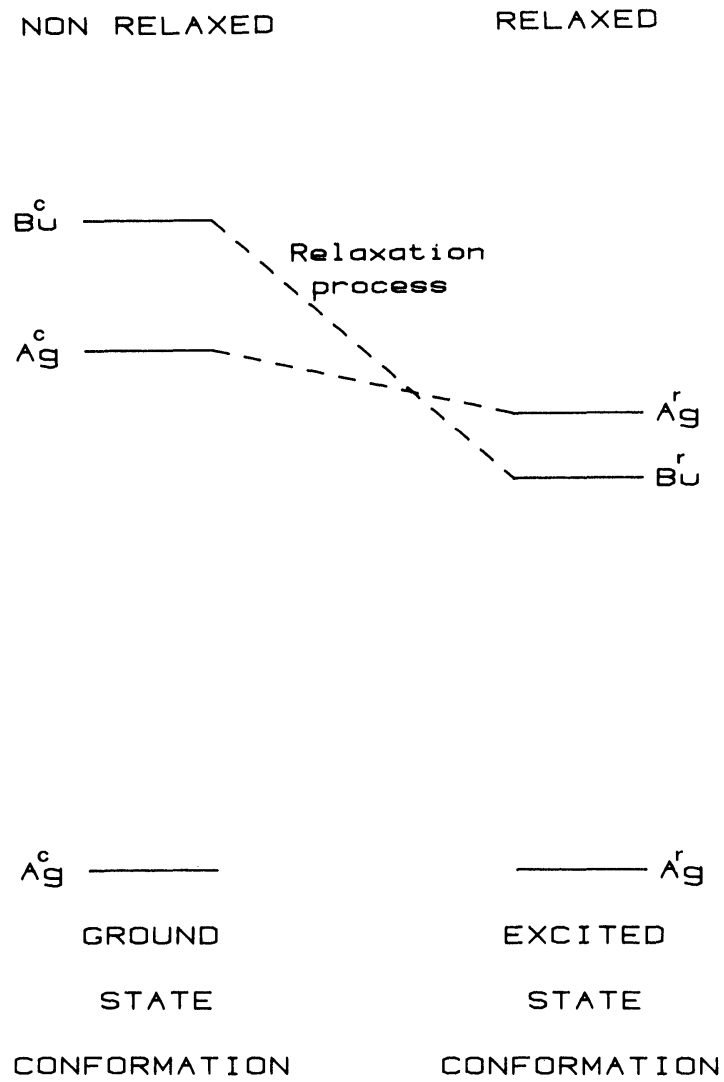

FIGURE 6 Illustrative diagram showing the level ordering dependence upon conformational change as explained in the text. 
electronic state, the DPB molecule exists in a certain stable conformation which embodies, in solution, coupling to the cage molecules of the solvent; to this stable conformation corresponds a well defined geometry of the super-molecule which minimizes, in the Born-Oppenheimer scheme, the electronic energy. This geometry corresponds to equilibrium values $\left(Q_{i}^{0}\right)$ of variables such as bond lengths, twist angles of the phenyl groups and of the polyenic bonds compatible with intramolecular and solvent contribution. In this ground state conformation of the super-molecule, the electronic Hamiltonian $H\left(Q^{0}\right)$ shows, for the first two excited singlet states, a specific level ordering with the lower level being of $A_{g}$ symmetry and the upper one of $B_{u}$ symmetry. So, after the one photon excitation step, the supermolecule is found electronically excited $E^{*}\left(Q^{0}\right)$ in an unstable nuclear conformation, due to electronic perturbation which causes forces $\left(\left\{\partial E^{*} / \partial Q_{i}\right\}_{Q_{i}^{0}}\right)$ on the conformation variables $Q_{i}$. Then, the supermolecule moves (relaxes) to a stable conformation $\left(Q_{0}^{*}\right)$ which cancels these forces $\left(\left\{\partial E^{*} / \partial Q_{i}\right\}_{Q_{0}^{*}}=0\right)$, causing appropriate changes to the active conformation variables of the supermolecule; for instance, these changes may occur in the twistings, in the bond lengths or the reorganization of the solvent molecules. In the next section we will discuss the exact nature and the efficiency of the relaxation channels leading to the stable supermolecular conformation in the excited electronic state. In this section, we want simply to point out that the possibility and the rate of relaxation of the excited molecule may be directly related to the molecular environment, in the sense that energy outflow, or energy inflow necessary to assist twisting energy barriers, for example, imply the existence of a lattice and the possibility to exchange energy with it. This may be exemplified in two limit cases where such a relaxation is not allowed: (i) the case of isolated, jet-cooled molecules in supersonic jets; (ii) the case of rigidized molecules in frozen solutions. In these two cases, respectively, intramolecular radiationless decay and decay via reorganization necessitating overflow energy, are hindered. Having in mind this prerequisite for reorganization mechanisms in the excited molecule, we want to show that our model of molecular dynamics is self consistent with all the experimental observations related to various stages of the supermolecular excitation. It may be sketched as follows: (i) in a molecular beam experiment, the lower singlet excited state, in the "cold" DPB conformation, is identified to be of $A_{g}$ symmetry, by two-photon absorption 
spectroscopy. So, even if the exciting radiation contains additional energy, above the resonance energy of $A_{g}$, to assist twisting, the excited molecular conformation $S_{1}^{*}$ does not change, remains of $A_{g}$ symmetry, as it has been demonstrated by Zewail and co-workers. ${ }^{12}$ Therefore, since the "cold" conformation does not change in the excited state, we conclude naturally that the optically excited state, by virtue of the vertical transition scheme, preserves the conformation of the ground state supermolecule, as discussed above. (ii) Our own experiments of one photon absorption spectra of excited DPB molecules in rigid solution, of Figures 3-4, provide the possibility to analyze further stages of the optically prepared state, since we are probing secondary events during the whole lifetime of the excited molecule. Indeed, solvent assisted reorganization mechanisms are frozen and they cannot compete with intramolecular decay rates in the cold conformation, cf. Figure 6: Just after one photon excitation (using third harmonic) in the $B_{u}$ manifold and fast vibrational relaxation in the cold conformation, only the vibrationless $B_{u}$ level is found populated. Therefore, the absorption band $B$ of excited DPB molecules appearing at $16000 \mathrm{~cm}^{-1}$ after the optical excitation step, must be assigned to transitions from the upper state $B_{u}$ to further excited states $S_{n}^{*}$, according to one photon selection rules. This is consistent with level ordering demonstrated in a cold conformation in (i), cf. left part of Figure 6. As time passes, intramolecular internal conversion in the cold conformation, with phonon emission to the lattice, populates state $A_{g}$ at the expense of state $B_{u}$. This explains the delayed appearance $(t>50 \mathrm{ps})$ of band A which we may naturally assign to transitions from state $\boldsymbol{A}_{\mathrm{g}}$ to further excited states $S_{n}^{*}$, according to one photon transition selection rules. At longer times $(t \sim 100 \mathrm{ps})$, decay of state $B_{u}$ to state $A_{g}$ is completed, the absorption spectrum originates only from population of state $A_{g}$, cf. Figure 3, with a decay time of 1.2 ns. Taking into account the dynamics of the excited molecule and the exciting pulse width of $25 \mathrm{ps}$, we estimated a value of $20 \mathrm{ps}$ for $B_{u}^{c} \rightarrow^{k^{\prime}} A_{g}^{c}$, the internal conversion rate, in the cold conformation. This value of $20 \mathrm{ps}$ is in very good agreement with rate values reported for other diphenyl polyenes, such as DPH and DPO. ${ }^{15}$ Concerning our experiments, it is worth mentioning that the decay time of $1.2 \mathrm{~ns}$ we measured for band $\mathrm{A}$, and a corresponding high quantum yield, ${ }^{5,6}$ seems incompatible with the radiatively forbidden character of state $A_{g}$. The strong radiative decay of state $A_{g}^{c}$ indicates that, owing to the small energy 
gap between states $A_{g}^{c}$ and $B_{u}^{c 1,3}$ the solvent enhances the vibronic coupling between these two states. The importance of the solvent contribution to the mixing of the two states may be evaluated when we compare the value of $1.2 \mathrm{~ns}$ in glassy solutions to the value of $10 \mathrm{~ns}$ observed for decay of state $A_{g}^{c}$ in a molecular beam experiment. ${ }^{12}$ Such solvent effects in vibronic mixing have been theoretically evaluated to account for photophysics in polyenic compounds. ${ }^{16}$ (iii) New channels of conformational dynamics are revealed in the absorption spectra of excited DPB molecules in liquid solutions. The time development of the absorption spectra are completely different, cf. Figures 1-2; this may be rationalized in our model as follows: A conformation reorganization channel is now competing with internal conversion in the cold conformation, we discussed in (ii); it leads to the following dynamics pattern: Just after one photon transition state $B_{u}^{c}$ is populated, cf. left part of Figure 6, fast conformation reorganization is energetically possible with rate constant $k(T)$ and leads to an energy trapped level inversion, with state $B_{u}$ remaining the only populated, cf. right part of Figure 6. Thus, during the first 50 picoseconds after excitation, we observe only band B as shown in Figure 2. A straightforward conclusion is that the conformation reorganization rate constant $k(T)$ is faster than internal conversion in the cold conformation we measured in (ii). $k(T)$ is probably faster than $10^{11} \mathrm{sec}^{-1}$ cannot give a more accurate indication with our experimental set-up. At longer times, the intensity of band A, which appears delayed with a larger rise-time in Figure 2, reaches a plateau depending strongly on the temperature. Self consistently band A may be attributed to state $A_{g}$ thermally populated at the expense of state $B_{u}$. Indeed, owing to their small energy gap $\Delta E$, thermal exchange of population cannot be neglected in liquid solutions where, at long times $t>100 \mathrm{ps,}$ a large number of thermal events occur; populations of the two states reach an equilibrium, with an asymptotic ratio $N\left(A_{g}\right) / N\left(B_{u}\right)=$ $\exp (-\Delta E / k T)$. At still longer times, the two states decay with the same lifetime as it is illustrated in Figure 2. Therefore, we assume the following scheme I with notations of Figure 6:

$$
\begin{aligned}
& S_{0}\left(A_{g}^{c}\right)+h v_{\mathrm{exc}} \stackrel{\begin{array}{c}
\text { Gaussian pulse } \\
25 \mathrm{ps} \text { width }
\end{array}}{\longrightarrow} S_{1}^{*}\left(B_{u}^{c}\right) \text { excitation step } \\
& S_{1}^{*}\left(B_{u}^{c}\right) \stackrel{K_{r}<10 \mathrm{ps}}{\longrightarrow} S_{1}^{*}\left(B_{u}^{r}\right) \quad \begin{array}{l}
\text { level inversion } \\
\text { via reorganization }
\end{array}
\end{aligned}
$$




$$
\begin{aligned}
& S_{1}^{*}\left(B_{u}^{r}\right) \stackrel{k}{\longrightarrow} S_{2}^{*}\left(A_{g}^{r}\right) \quad \text { thermal population } \\
& S_{2}^{*}\left(A_{g}^{r}\right) \stackrel{k^{\prime}}{\longrightarrow} S_{1}^{*}\left(B_{u}^{r}\right) \quad \text { internal conversion } \\
& S_{1}^{*}\left(B_{u}^{r}\right) \stackrel{k_{1}}{\longrightarrow} S_{0}\left(A_{g}^{r}\right) \quad \begin{array}{l}
\text { radiative and } \\
\text { non-radiative transition }
\end{array} \\
& S_{2}^{*}\left(A_{g}^{r}\right) \stackrel{k_{2}}{\longrightarrow} S_{0}\left(A_{g}^{r}\right) \quad \begin{array}{l}
\text { radiative and } \\
\text { non-radiative transition }
\end{array}
\end{aligned}
$$

Assuming for the excitation pulse a gaussian shape with a width of 25 ps and decay times $1 / k_{1}=800$ ps and $1 / k_{2}=1.2 \mathrm{~ns}$, we have simulated the kinetics of the excited DPB molecule on the basis of scheme I. In doing so, we assumed the asymptotic form for the rates $k=$ $k^{\prime} \exp (-\Delta E / k T)$ with the value $1 / k^{\prime}=20 \mathrm{ps,}$ which is the one measured for internal conversion at low temperatures (cold conformation). The value $\Delta E=500 \mathrm{~cm}^{-1}$ is chosen for the energy gap, the measured value varies in the limits of 300 and $1000 \mathrm{~cm}^{-11,2,5}$ depending on the experimental conditions.

As shown on Figure 2 the agreement between experimental points and simulated curves, obtained on the basis of scheme $\mathrm{I}$, is excellent. A change of the value $\Delta E$, between the experimental limits, is not affecting our essential conclusion that the kinetics of scheme I are consistent with our observations at room temperature.

Our experimental data on the picosecond scale, reinforce the hypothesis on the level ordering of the excited states in solution at room temperature: the lower excited state is of $B_{u}$ symmetry. In addition, we bring evidence that, as for DPO molecules in solution, excited states of DPB molecules exchange populations thermally; an equilibrium is reached between relaxed conformations at $t>100 \mathrm{ps,}$ population of state $A_{g}^{r}$ being the weaker one. At intermediate temperatures, conformational exchange rates does not compete efficiently with intramolecular conversion in the cold conformation. Therefore, the excited molecules exist in the two conformations with corresponding exchange rates between four states as precised on Figure 6, with contributions of the conformations varying with temperature as shown on Figure 4.

To conclude, our picosecond observations bridge all the experimental conditions reported which are made consistent on the basis of 
a dynamical model assuming a very fast, solvent assisted, conformational exchange which inverts the level ordering of the first two excited states $A_{g}$ and $B_{u}$. However, the shape dependence on the intensity $I_{\text {exc }}$ of the exciting pulse has not received an explanation on the basis of our model. To this respect, our observations may be related to the work reported by Kliger and co-workers ${ }^{13}$ on the absorption spectra of excited solutions of DPH, DPO and DPB molecules in liquid solutions. For DPH and DPO, whose first excited state is of $A_{g}$ symmetry, these authors observed two bands: one red band near $15300 \mathrm{~cm}^{-1}$ and one blue band near $25000 \mathrm{~cm}^{-1}$. For DPB, whose lower excited state is of $B_{u}$ symmetry, they observed only one red band near $15300 \mathrm{~cm}^{-1}$, the absence of the blue band being interpreted as evidence of the $B_{u}$ symmetry of the lower excited state. Self consistency of the observations of Kliger and co-workers and our model predicts that, at low temperatures for which we assume no inverted levels in a "cold" conformation, one blue band should appear also for DPB. In fact, the spectral sensitivity of our experimental set-up is limited to wavenumbers smaller than $25000 \mathrm{~cm}^{-1}$, so that no such blue band may be detected. However, we may report indirect evidence for the existence of the blue band in the analysis of the dependence of absorption shape vs. the intensity $I_{\text {exc }}$ of the exciting beam, discussed for Figure 5 . Indeed, the spectral changes with $I_{\text {exc }}$ may be rationalized as follows: an increase of $I_{\text {exc }}$ leads to an increase of population of state $B_{u}$. This population increase may be tested, at a fixed time $t=100 \mathrm{ps}$, at which the excitation process is ended. During the excitation process, state $A_{g}$ is populated at the expense of state $B_{u}$ by internal conversion whose rate $\left(\sim 1 / 20 \mathrm{ps}^{-1}\right)$ may be compared to the excitation duration (25 ps). Therefore, transitions $S_{1}^{*}\left(A_{\mathrm{g}}^{c}\right) \rightarrow S_{n}^{*}$, which have a strong absorption near the wavelength of the exciting beam $\left(28328 \mathrm{~cm}^{-1}\right)$ may populate state $B_{u}$ via internal conversion $S_{n}^{*} \rightarrow B_{u}$. Then, at the fixed time $t=100 \mathrm{ps}$ after the excitation step, the population $N_{B_{u}}(t, T)$ may be given the following dependence on $I_{\text {exc }}$ :

$$
N_{B_{u}}(t, T)=a(t) I_{\mathrm{exc}}+c(t) I_{\mathrm{exc}}^{2}+b(t, T) I_{\mathrm{exc}}^{2}
$$

assuming for the quadratic change of the intensity of band $\mathrm{B}$, contributions, respectively, from two-photon transitions and from two steps, temperature dependent, transitions via state $A_{g}$ as illustrated in Figure 7. Therefore, the existence of the blue band of DPB associated to the 


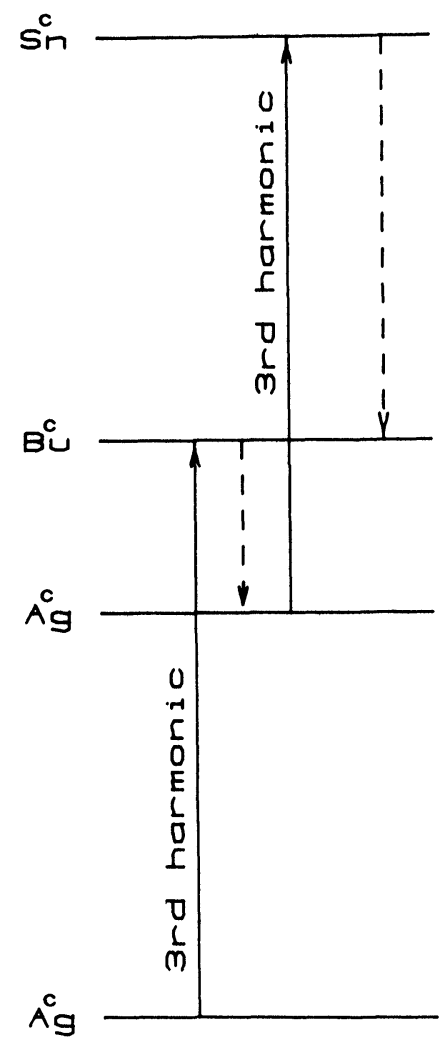

FIGURE 7 Illustrative diagram showing the influence of two-steps process on the $B_{u}$ state population at low temperature.

absorption transitions from state $A_{g}$, is revealed by our experiments; they support the $\mathrm{Ag}$ character assignment of the lower excited singlet state in frozen solutions and the self consistency of our model based on excited conformational dynamics. These qualitative points being established, we procede further to a quantitative discussion on the nature of the conformational dynamics.

\section{DISCUSSION AND CONCLUSION}

In this section we discuss on the exact nature of the relaxation channels which are responsible for conformational exchange in the excited 
supermolecular state and for deactivation to the ground state. As illustrated on Figure 8, DPB is a flexible molecule with important conformational solvent assisted changes that may originate in two parts of the molecule: One is located in the polyenic chain whose twisting of double or single bonds may lead to various conformations measured by the angle $\theta_{e}$.

The other part concerns the phenyl groups where ring twisting may create new conformations measured by the angle $\theta_{p}$, cf. Figure 8 .

The effects of twisting in the polyenic chain have been extensively discussed for the excited DPB molecules, mainly by analogy with $t$-stilbene molecule which undergoes very active photoisomerization. Along these lines, Zewail and co-workers ${ }^{12}$ have explained the photophysical behavior of DPB, using the $t$-stilbene photoisomerization potential curve. Also, Kliger and co-workers assumed, ${ }^{13}$ as a work hypothesis, double bond twisting in the polyenic chain in order to account for discrepancies between observed spectra and theoretical calculations results. Furthermore, these authors synthetized and studied a rigidized DPB derivative (the HNN compound) where twisting in the polyenic chain is totally hindered. ${ }^{17}$ From the analysis of this compound, they concluded that DPB, in state $B_{u}$, is planar and that the work hypothesis of twisting in the polyenic chain has to be rejected. ${ }^{17}$

As a matter of fact, twisting in the polyenic chain does not appear as the most efficient mechanism to account for very fast conformational dynamics in the excited state with subsequent spectral changes observed. Indeed, twisting in the chain occurs in a time of the order of one hundred of picoseconds. ${ }^{11}$ Compared to the reorganization rates of 10 picoseconds we estimated for the excited DPB molecule, torsions in the polyenic chain are too slow to contribute in the fast spectral changes we observed. A more compatible mechanism with our experimental data, seems to be that of phenyl rings twisting,

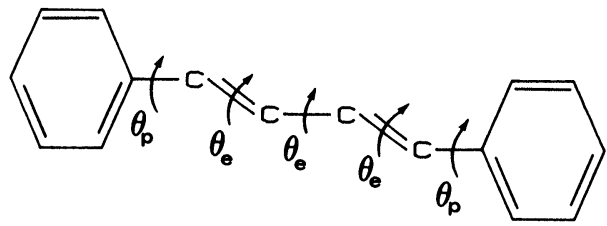

FIGURE 8 1,4-diphenyl-butadiene with angles characterizing conformational changes. 
although this mechanism has received little attention, being only mentioned by Kliger and co-workers. ${ }^{17}$ Phenyl twisting is generally a fast, non-radiative process which competes efficiently with radiative process. For example, it has been shown for triphenyl methane dyes, that phenyl twisting totally quenches the fluorescence at room temperature, ${ }^{18,19,20,21}$ its rate is evaluated to be in the range of $10 \mathrm{ps}^{18}$ Moreover the twisting rate is very sensitive to the local properties of the lattice, such as temperature and viscosity in non-polar solvents. ${ }^{19,20}$ For the above mentioned triphenyl methane dyes, phenyl twisting is quenched at low temperatures. ${ }^{19,20}$ Thus efficiency of such a mechanism requires specific conditions related to the energy gap between levels $B_{u}$ and $A_{g}$ and to the energy barriers overflow in the path leading to level inversion by phenyl (twisting), with possible solvent assistance along these states of conformational exchange. More precisely, the energy of state $B_{u}$ must be more sensitive to the twist than energy of state $\boldsymbol{A}_{\mathrm{g}}$. To show this possibility, we calculated by quantum chemistry calculation technics the influence of phenyl twist on the energy levels of state $B_{u}$ and state $A_{g}$. The theoretical procedure used and some other implications are described in Appendix I. The principal results of our calculations are illustrated on Figure 9. They show that the relative energy change of state $B_{u}$ is much more sensitive to phenyl twist than that of state $A_{g}$. In addition, these calculations indicate that small changes in the twist angle are sufficient to cause level inversion. Comparison of our experimental and calculated results allows us to give a reasonable picture of the various stages of the molecular excitation: (i) in the ground state of the supermolecule, the phenyl rings are twisted out of the molecular plane, as it has been already observed for the diphenylnaphthalene, ${ }^{22}$ for instance, while the polyenic chain is in an all-trans conformation. The electronic Hamiltonian shows that the lower singlet excited state is $\boldsymbol{A}_{\boldsymbol{g}}$. (ii) After, one photon excitation, the excited state is the upper state $B_{u}$, cf. Figure 10. (iii) At room temperature, fast phenyl twist leads to level inversion, with energy trapped $B_{u}$ state becoming the lower one, in a more planar conformation, cf. Figure 10. Such a conformation change implies solvent contribution, the simplest of which is overflow energy provided by the lattice. Hence, lowering the temperature (or increasing viscosity) may slow down, even hinder, the effects of fast conformational exchange due to phenyl mobility. This model prediction is in full agreement with our experimental observations at low temperatures. 


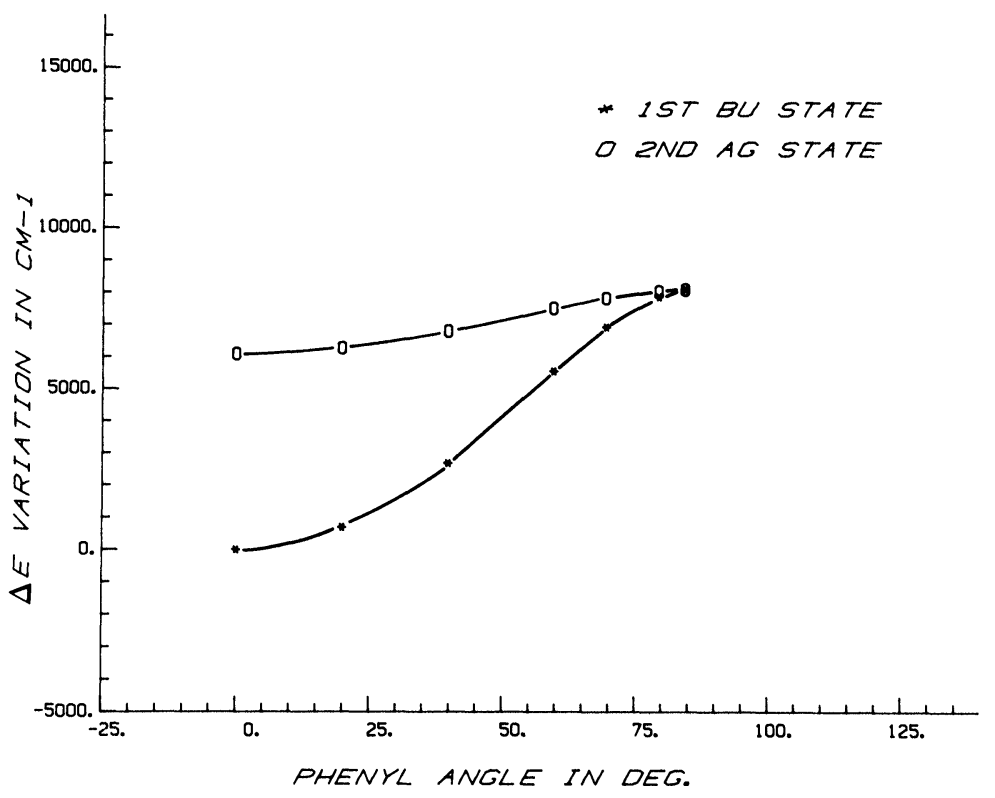

FIGURE 9 Relative energy $\Delta E$ variations of the first calculated $B_{u}$ state and of the second calculated $A_{g}$ state as a function of phenyl twist angle $\left(\theta_{p}\right)$. Calculation is made according to theoretical methods explained in Appendix I.

To summarize the optically prepared $B_{u}$ state is perturbed by various dynamic channels affecting in specific ways the spectral distribution of the absorption spectrum and its decay to the ground state:

(i) Very fast oscillations of the order of $10 \mathrm{ps}$ and environment assisted, between two excited conformations induce fast spectral changes. They are controlled by the phenyl mobility, the energy trapped state is $B_{u}$ in a planar conformation;

(ii) Intramolecular, radiative and non-radiative, decay to the planar ground state;

(iii) Non-radiative decay to the ground state (chain twisted conformation), via environment assisted twist in the polyenic chain, analogous to the decay path observed in excited $t$-stilbene or organic dyes.

The oscillation rate (i) of the order of 10 ps we measured at room temperature, is mainly related to spectral changes observed in the absorption shape of excited molecules. The mechanisms (ii) and (iii) 


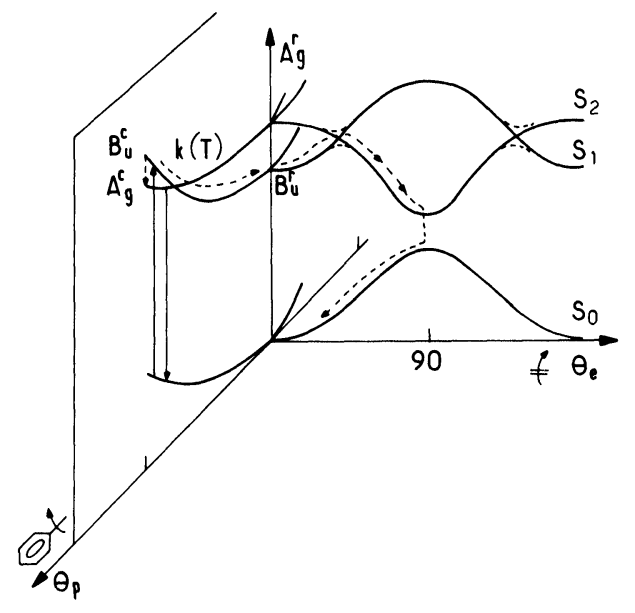

FIGURE 10 Proposed diagram to account for the photophysical behaviour of DPB.

account for decay rates. These mechanisms have been extensively studied by Fleming and co-workers who measured their decay rates to the ground state and found values in the range of $100 \mathrm{ps}$.

\section{CONCLUSION}

The work presented in this paper sheds new light on the photophysical behavior of the DPB molecule in solution. Mainly, we bring evidence that fast conformational exchange, due to the phenyl groups mobility, occurs in the excited state of DPB in liquid solution; it causes inversion of level ordering of the first singlet excited states. This conformation exchange and level inversion are hindered at low temperatures where state $A_{g}$ remains the lower one. Our photophysical model, illustrated on Figure 10, allows us to bridge experimental observations in a large variety of conditions such as "cold" DPB molecules, in a molecular beam, to flexible DPB molecules in a liquid solution. In the present work, we considered only effects of temperature in non-polar solvents. Experiments in polar solvents should allow us to refine our knowledge on the photophysical properties of the excited conformation of this molecule. Such results will be reported in a forthcoming paper. 


\section{APPENDIX I}

\section{Theoretical procedure and results}

To calculate energy and symmetry of singlet states involved in the photodynamics, we used an SCF-LCAO-MO-CI calculation in the approximation of Pariser-Parr-Pople. ${ }^{23,24}$

Bicentric, bielectronic integrals were used in the formulation of Pariser and Parr. ${ }^{23}$ The parameters, bond lengths and angles, used are given in Table I.

To calculate the state dependence upon twist angle $\theta_{p}$ of phenyl ring we used the following procedure. The molecular resonance integral $\beta_{c c}$ between carbon atoms of phenyl rings and polyenic chain, was given by the following relation ${ }^{25,26}$

$$
\beta_{c c}=\beta_{0} \cos \theta \quad \text { with } \beta_{0}=-2.32 \mathrm{eV}
$$

At each angle $\theta_{p}$ the DPB geometry was optimized by successive iterations on the bond length $\mathrm{C}-\mathrm{C}$ between phenyl ring and polyenic chain using the following relation between bond order $P_{p q}$ and bond length $l_{c c \cdot}{ }^{27}$

$$
l_{c c}=1.52-0.19 P_{p q}
$$

\section{TABLE I}

Parameters used for theoretical procedure: $Z_{\text {eff }}$ : Slater effective nuclear charge of carbon atom (allowing $\gamma_{p q}$ calculation belong Pariser method). $I$ : ionization potential of trigonal carbon atom. $A$ : electronic affinity of trigonal carbon atom. $W_{2 p}=-I=$ atomic valence state ionisation potential. $\gamma_{c c}=I-A=$ Coulomb repulsion integral of carbon atom

\begin{tabular}{llc}
\hline$Z_{\text {eff }}=3.25$ & $\gamma_{c c}=I-A=11.13 \mathrm{eV}$ & $l_{c c}$ aromatic $=1.40 \AA$ \\
$W_{2 p}=-11.16 \mathrm{eV}$ & $l_{c c}$ (angle bond) $=1.48 \AA$ & $\theta_{c c}=120^{\circ}$ \\
$\beta_{c-c}^{c}=-2.32 \mathrm{eV}$ & $l_{c c}$ (double bond $)=1.34 \AA$ & \\
\hline
\end{tabular}

TABLE II

Theoretical results. The numbers for singly excited configurations one as in Ref. 3 where numbers from 0 to 8 designate occupied orbitals and numbers 9 to 16 designate unoccupied orbitals in the ground state

\begin{tabular}{cccc}
\hline State & Symmetry & $\begin{array}{c}\text { Energy in } \mathrm{cm}^{-1} \\
\text { (above ground state) }\end{array}$ & $\begin{array}{c}\text { Principal singly } \\
\text { excited configurations }\end{array}$ \\
\hline 1 & $B_{u}$ & 32580 & $8-9$ \\
4 & $A_{g}$ & 39300 & $(8-10)+(7-9)$ \\
\hline
\end{tabular}




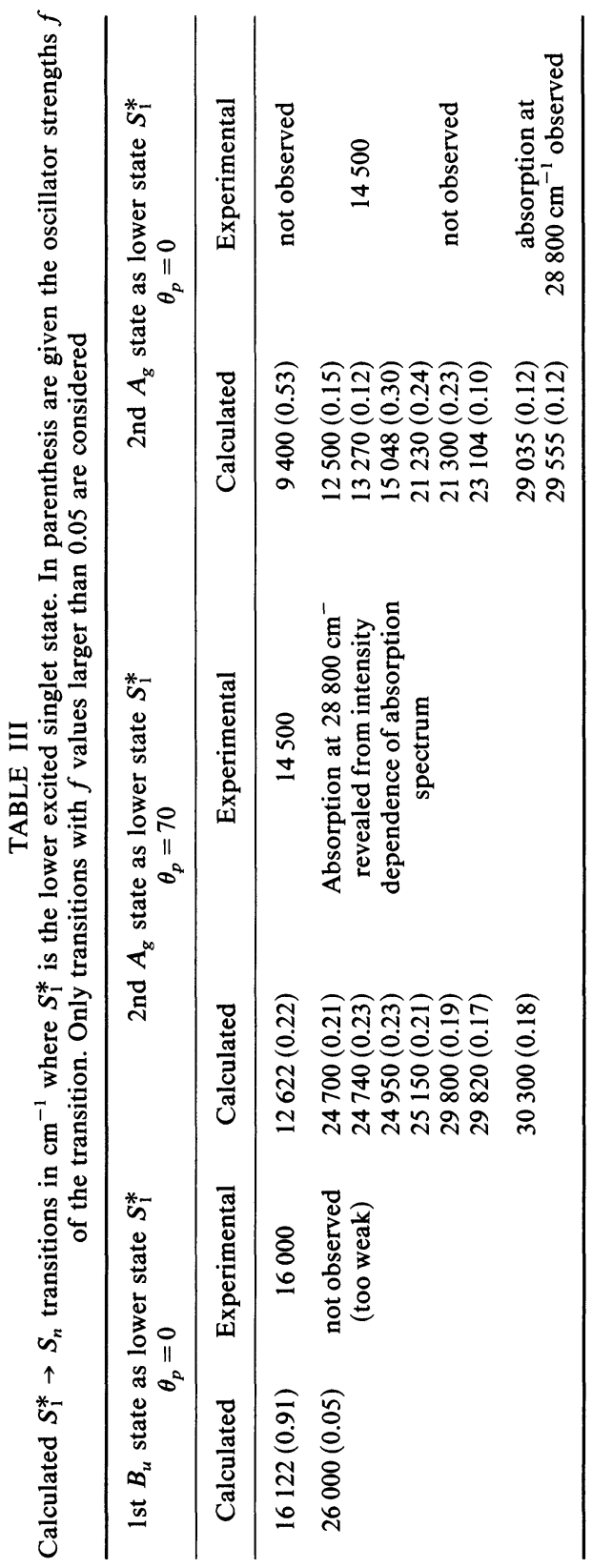


All singly excited configurations were used in the theoretical procedure and the results are described in Table II, Table III and Figure 9.

Table II shows that using only singly excited configurations does not affect the relative ordering of the two concerned states compared to methods using doubly excited configurations. ${ }^{3,13}$ Only the energy of the states is overestimated.

The failure of theoretical methods to correctly calculate the relative order of DPB singlet excited state may be perfectly explained if we take into account geometry factors. All previous calculations ${ }^{3,13}$ were made using a planar or quasi-planar geometry. Figure 9 illustrates that introduction of phenyl twisting may give correct ordering even considering the second calculated state as the $A_{g}$ state observed experimentally. ${ }^{3}$ Indeed, introduction of doubly excited configurations lowers the $A_{g}$ states of approximately $3000 \mathrm{~cm}^{-1}$ with respect to $B_{u}$ states. ${ }^{3,13}$ Then, such a lowering should introduce crossing between $A_{g}$ and $B_{u}$ for $\theta_{\mathrm{p}}$ of the order of $60^{\circ}$, which should give a $\theta_{p}$ value of $70^{\circ}$ where level ordering should be correct. Such a value is in good agreement with $74^{\circ}$ calculated value of equilibrium for $\mathrm{HHN}$ in the ground state using MM2 method. ${ }^{17}$

Table III shows that experimental results concerning $S_{1} \rightarrow S_{n}^{*}$ excited absorption spectra are correctly reproduced by our theoretical calculation taking into account, in planar geometry, the $B_{u}$ state as the lower state or, in the twisted conformation, the 2nd calculated $\boldsymbol{A}_{\boldsymbol{g}}$ state as the lower state. Moreover, like in 1,4-diphenyl-naphthalene, planar geometry $\left(\theta_{p}=0\right)$ and 2 nd calculated $A_{g}$ state as lower state is unable to fit experimental results showing that twisted conformation is a reasonable and plausible hypothesis.

\section{References}

1. R. L. Swofford and W. M. McClain, J. Chem. Phys. 59, 5740 (1973).

2. R. L. Swofford and W. M. McClain, Rev. Sci. Instr. 46, 246 (1975).

3. J. A. Bennett and R. R. Birge, J. Chem. Phys. 73, 4234 (1980).

4. J. B. Birks, G. N. R. Tripathi and M. D. Lumb, Chem. Phys. 33, 185 (1978).

5. S. P. Velsko, G. R. Fleming, J. Chem. Phys. 76, 3553 (1982).

6. D. J. S. Birch, R. E. Imhof, Chem. Phys. Lett. 88, 243 (1982).

7. K. M. Keery and G. R. Fleming, Chem. Phys. Lett. 93, 322 (1982).

8. P. C. Alford and T. F. Palmer, J. Chem. Soc. Faraday Trans. 79, 433 (1983).

9. P. C. Alford and T. F. Palmer, Chem. Phys. Lett. 86, 248 (1982).

10. D. J. S. Birch and J. B. Birks, Chem. Phys. Lett. 38, 432 (1976).

11. R. M. Hochstrasser, Pure and Appl. Chem. 52, 2683 (1980). 
12. J. F. Shepanski, B. W. Keelan and A. H. Zewail, Chem. Phys. Lett. 103, 9 (1983).

13. R. A. Goldbeck, A. J. Twarowski. E. L. Russel, J. K. Rice, R. R. Birge, E. Switkes and D. S. Kliger, J. Chem. Phys. 77, 3319 (1982).

14. C. Rullière, A. Declémy and Ph. Pée, Rev. Phys. Appl. 18, 347 (1983).

15. T. C. Felder, K. J. Chai and M. R. Topp, Chem. Phys. 64, 175 (1982).

16. G. Hug and R. S. Becker, J. Chem. Phys. 65, 55 (1976).

17. W. A. Yee, J. S. Horwitz, R. A. Goldbeck, C. M. Elnterz and D. S. Kliger, J. Phys. Chem. 87, 380 (1983).

18. D. Magde and M. W. Windsor, Chem. Phys. Lett. 24, 144 (1974).

19. G. Oster and Y. Nishijima, J. A.C.S. 78, 1581 (1956).

20. Th. Förster and G. Hoffman, Z. Physik. Chem. NF 75, 63 (1971).

21. V. Sundstrom, T. Gillbrö and H. Bergström, Chem. Phys. 73, 439 (1982).

22. E. L. Russel, A. J. Twarowski, D. S. Kliger and E. Switkes, Chem. Phys. 22, 167 (1977).

23. R. Pariser and R. G. Parr, J. Chem. Phys. 21, 767 (1953).

24. J. A. Pople, Trans. Faraday Soc. 49, 1375 (1953).

25. R. G. Parr and B. L. Crawford, J. Chem. Phys. 16, 526 (1948).

26. M. J. S. Dewar, J. Am. Chem. Soc. 74, 3345 (1952).

27. M. Roche, F. d'Amato and M. Benard, J. Mol. Struct. 9, 177 (1971). 